Musées, Patrimoine et Culture scientifiques et techniques

$122 \mid 2009$

mars - avril 2009

\title{
La problématique du dégraissage des squelettes
}

\section{Gwenaël Lemoine et Élodie Guilminot}

URL : http://journals.openedition.org/ocim/283

DOI : $10.4000 /$ ocim.283

ISSN : 2108-646X

Éditeur

OCIM

Édition imprimée

Date de publication : 1 mars 2009

Pagination : 12-18

ISSN : 0994-1908

Référence électronique

Gwenaël Lemoine et Élodie Guilminot, "La problématique du dégraissage des squelettes », La Lettre de l'OCIM [En ligne], 122 | 2009, mis en ligne le 01 mars 2011, consulté le 30 avril 2019. URL : http:// journals.openedition.org/ocim/283; DOI : 10.4000/ocim.283 


\section{La problématique du dégraissage des squelettes}

\section{Gwenaël Lemoine et Élodie Guilminot *}

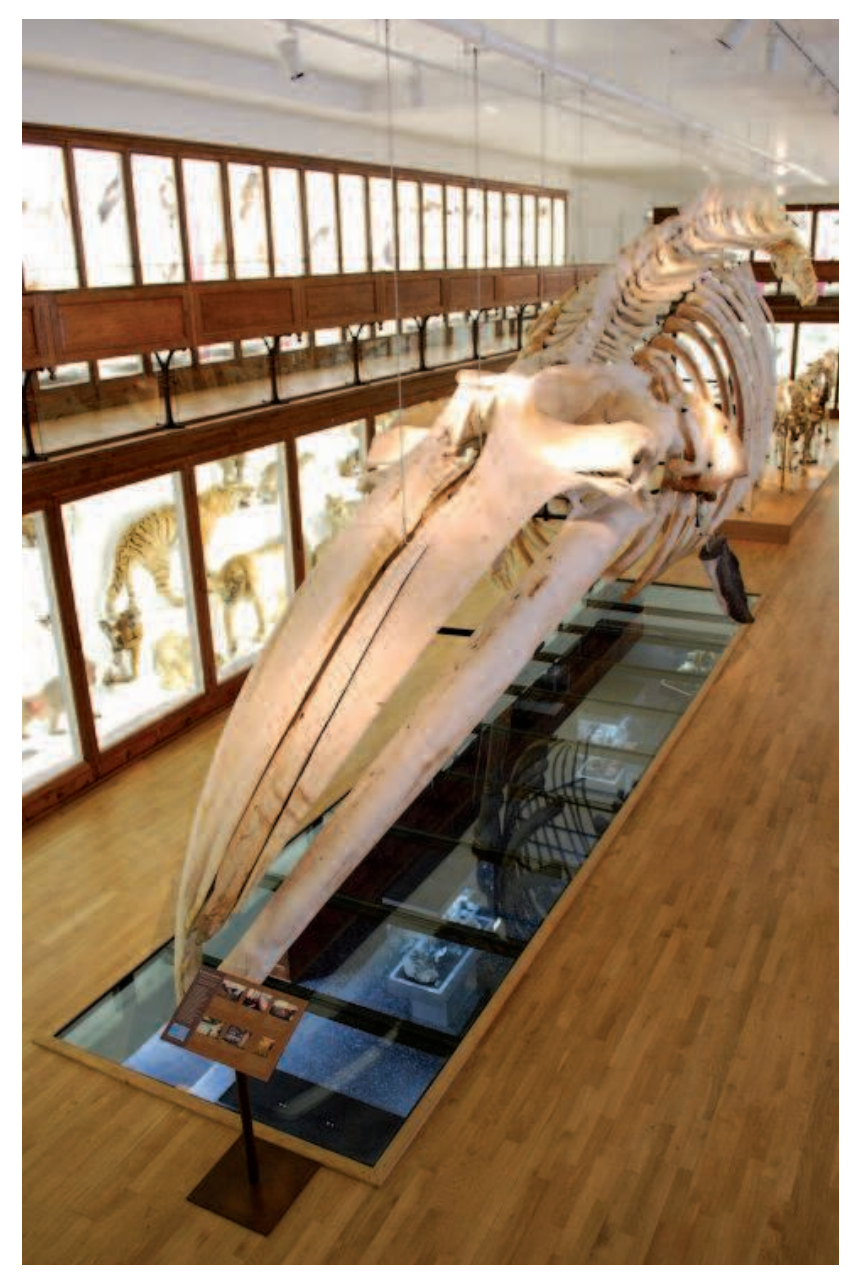

Le squelette de rorqual commun (Balaenoptera physalus) exposé au muséum d'Histoire naturelle de Nantes ○ Muséum d'Histoire naturelle de Nantes/Patrick Jean

* Gwenaël Lemoine est conservateur-restaurateur à Arc'Antique. Élodie Guilminot est ingénieur de recherche à Arc'Antique. arcantique.organique@orange.fr
Les techniques actuelles de dégraissage des collections ostéologiques ne donnent pas toujours entière satisfaction et de nombreux muséums d'Histoire naturelle rencontrent ce problème de graisse suintant à la surface des os - notamment sur les ossements poreux et gras des mammifères marins - parfois des décennies après la préparation du squelette : les auteurs rendent compte d'une étude consistant à trouver une alternative aux solvants chimiques toxiques pour le dégraissage du squelette de baleine du muséum d'Histoire naturelle de Nantes.

Le laboratoire Arc'Antique a été sollicité par le muséum d'Histoire naturelle de Nantes pour le traitement du squelette d'un rorqual commun (Balaenoptera physalus), couramment appelé baleine, exposé depuis 1995. Malgré un traitement réalisé au début des années 1990, par macération puis immersion dans une solution à base de trichloréthane et de formol, les os présentent des dépôts de matière grasse brune suintant et dégageant une odeur rance aux températures estivales. La présence de graisses dans l'os et à sa surface pose deux problèmes majeurs : d'une part du point de vue esthétique (coloration brune) et d'autre part pour la conservation de l'os. La graisse favorise le développement de microorganismes et son évolution chimique (acidification du milieu, réticulation des protéines de l'os et dégradation du collagène) peut altérer l'os ${ }^{(1)}$. Un traitement de dégraissage est donc de nouveau nécessaire pour la conservation à long terme du squelette. 
Un sondage, effectué auprès des professionnels des collections d'Histoire naturelle sur les méthodes de préparation et de dégraissage des squelettes, nous a fait prendre conscience du caractère universel et complexe de cette problématique de traitement des os gras. Il ne concerne d'ailleurs pas uniquement les cétacés, les os des mammifères d'élevage ou de zoo s'avèrent être également très gras, car richement nourris dans des conditions d'exercices limités. Comme le dégraissage n'est pas toujours mené à cour, faute de temps, de moyens mais surtout de méthodes de contrôle, l'apparition de graisses à la surface des os est fréquente dans les années qui suivent la préparation du squelette, ceci quelle que soit la méthode utilisée pour le dégraissage. De nombreux musées se retrouvent dès lors confrontés à un suintement de graisse en surface de leurs collections, anciennes ou récentes.

\section{Une démarche scientifique}

L'intervention d'Arc'Antique s'inscrit dans la déontologie et le respect des principes de la conservationrestauration : examen de l'objet, documentation du travail, respect de l'intégrité physique et scientifique de l'objet, maîtrise et contrôle du traitement, respect des normes d'hygiène et de sécurité en vigueur actuellement. Afin de choisir un traitement adapté et efficace, il est nécessaire de bien connaître la nature du substrat - l'os et son état de conservation, les graisses à extraire et leur degré de dégradation. Cette connaissance s'applique également aux méthodes et produits utilisés, à leur action sur les graisses et leur impact sur l'os (2) (3) (4). Un tel projet nécessite une approche pluridisciplinaire qui associe scientifiques, préparateurs ostéologiques, restaurateurs et conservateurs.

\section{L'os gras, un matériau complexe}

L'os est constitué d'une partie organique, matrice de fibres de collagène rigidifiée par un remplissage serré de cristaux d'hydroxylapatite $\left(\mathrm{Ca}_{10}\left(\mathrm{PO}_{4}\right)_{6-\mathrm{x}}\left(\mathrm{CO}_{3}\right)_{\mathrm{x}}\right.$ $\left.(\mathrm{OH})_{2}\right)^{(5)}$. Tandis que l'apatite assure la tenue mécanique, le collagène apporte à l'os sa flexibilité ; en son absence, l'os devient friable. La préservation de ces deux constituants est donc indispensable pour la conservation à long terme de l'os.

Bien que légers et spongieux, les os des baleines sont relativement solides grâce à une couche externe, le périoste, plutôt fine et fibreuse mais dure et compacte. La partie interne de l'os, la «spongiosa ", est constituée d'un réseau de cavités très riches en graisse (6).
Les graisses contenues dans les os de baleine sont principalement des triglycérides (7), constitués d'acides gras saturés à 12-18 atomes de carbone, solides à température ambiante et d'acides gras insaturés à 16-20 carbones, liquides à température ambiante.

\section{Identification des graisses présentes dans le squelette du rorqual du muséum de Nantes}

En premier lieu, il est nécessaire d'identifier précisément les graisses présentes dans le squelette (triglycérides, phospholipides, stérols ...) et leur état de dégradation (en acides gras libres ou combinés au glycérol). Ces données sont indispensables pour établir le constat d'état du squelette et sélectionner un traitement de dégraissage efficace.

Les graisses prélevées au scalpel sont analysées par chromatographie. N'ayant pas accès à cette technique, Arc'Antique a fait appel au laboratoire Nicolas Garnier. Les résultats, obtenus par chromatographie en phase gazeuse, montrent que les graisses prélevées à la surface du squelette de rorqual contiennent principalement des acides gras saturés à 14,16 et 18 atomes de carbone. Les acides gras insaturés détectés dans l'huile native ${ }^{(8)}$ de la baleine ne sont plus présents dans les graisses prélevées à la surface du squelette de rorqual (9). Des analyses complémentaires effectuées par chromatographie en couche mince par

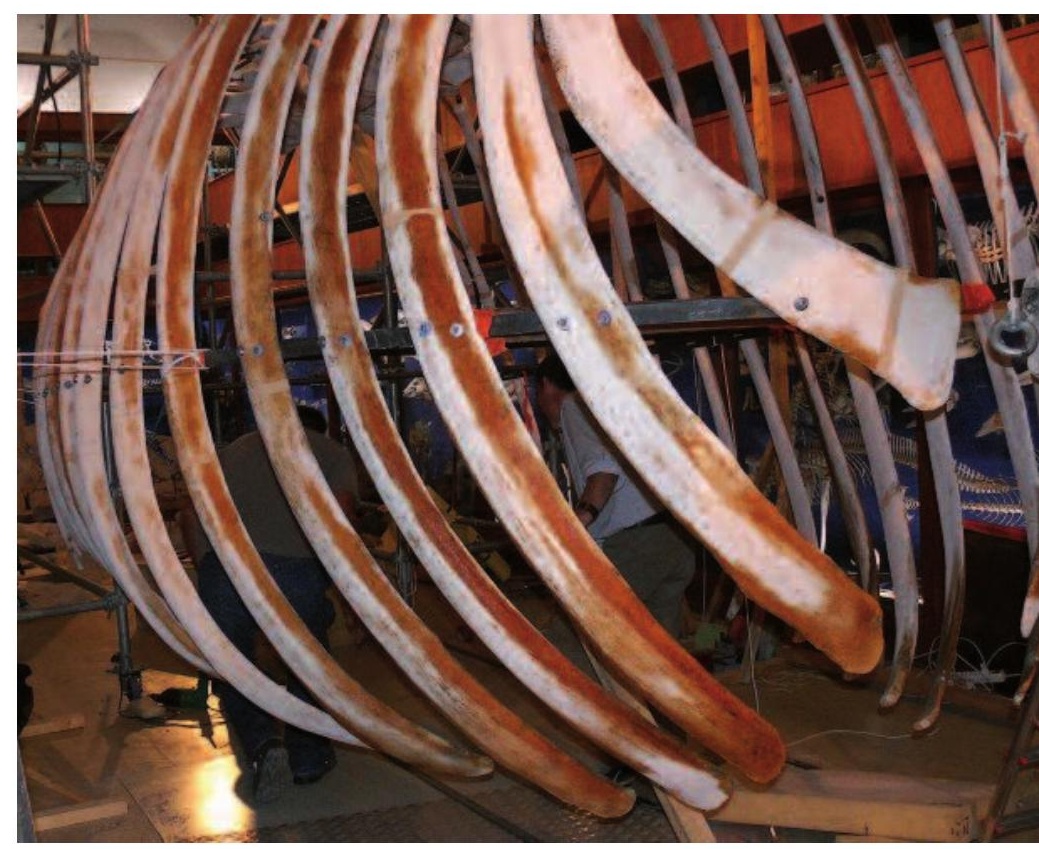

Le dégraissage, réalisé dans les années 1990, est insuffisant: les os suintent de graisse brune à l'odeur rance. Les vertèbres, côtes et omoplates sont les plus touchées. ๑) Muséum d'Histoire naturelle de Nantes/Patrick Jean 
l'IUT Génie Biochimie de Laval ont permis de déterminer l'état de dégradation de ces graisses : les triglycérides sont totalement dégradés en acides gras.

\section{Des graisses dégradées}

En effet, depuis la mort de l'animal, divers facteurs ont entrainé la transformation de ces graisses. Le traitement aqueux auquel la carcasse du rorqual a été soumise initialement, par immersion et jets d'eau à forte pression, a probablement entrâné une pénétration d'eau au cœur des os, favorisant ainsi l'hydrolyse des graisses. Puis au cours de l'exposition, les fluctuations d'humidité relative ont aussi pu provoquer une hydrolyse des graisses résiduelles. Les graisses ainsi hydrolysées sont communément appelées savons d'acides gras. Par ailleurs, une oxydation des acides gras semble inévitable : en effet, libres comme combinés (ce qui est le cas dans les triglycérides), les acides gras s'oxydent spontanément à l'air, l'auto-oxydation étant plus forte pour les molécules très insaturées et plus rapide lorsqu'il y a accès direct à l'oxygène. L'oxydation provoque le rancissement des huiles et des graisses : la formation de peroxydes sous l'effet de l'oxygène engendre, par rupture de chaînes, la production de radicaux libres, la réticulation des lipides et la production d'aldéhydes, produits volatiles de faible masse moléculaire et responsables de l'odeur, ainsi que d'acides, toxiques.

Avec l'oxydation des graisses, la partie organique de l'os risque d'être à son tour endommagée. Les radicaux libres ainsi formés entraînent des transformations des protéines dont une dégradation du collagène.

\section{Impact du traitement sur la conservation de l'os}

Avant d'envisager un traitement quel qu'il soit, il faut considérer l'impact inévitable sur les composants de l'os, que l'on cherche à conserver. Une méconnaissance du substrat et des produits de dégraissage utilisés peut mener à des dégâts irréversibles. Toute modification de la structure osseuse (altération de la fraction organique ou de la partie minérale) et de ses propriétés entraînerait une fragilisation structurelle de l'os. Les traitements de dégraissage peuvent aussi induire une altération des composants des os susceptibles de gêner leur étude scientifique, en biaisant l'exploitation des données.

\section{Techniques actuelles de dégraissage}

Dans la littérature, la description de la préparation des squelettes ne dissocie pas toujours la phase d'écharnage de celle du dégraissage du squelette (10) (11). Ces données bibliographiques ont été complétées et mises à jour grâce à un questionnaire envoyé par Arc'Antique en 2007 auprès de la communauté des préparateurs ostéologiques, taxidermistes et conservateurs confrontés à cette problématique de squelette gras.

La technique la plus utilisée reste la macération en solution aqueuse dont l'efficacité est due à l'action des bactéries. La température des bains peut varier de 35 à $60-70{ }^{\circ} \mathrm{C}$, voire jusqu'à $100^{\circ} \mathrm{C}$ (il s'agit alors de « cuisson»). Divers additifs sont ajoutés dans les solutions aqueuses :

- des tensioactifs (détergents favorisant la dissolution des graisses),

- des enzymes (principalement des protéases, telles que la papaïne ou la neutrase),

- du peroxyde d'hydrogène $\left(\mathrm{H}_{2} \mathrm{O}_{2}\right)$ qui permet de dissoudre la graisse et de blanchir les os,

- de la soude $(\mathrm{NaOH})$ ou de la potasse $(\mathrm{KOH})$ pour saponifier les graisses.

Lorsque la macération est insuffisante pour dégraisser les os, ce qui est le cas pour les squelettes gras tels que celui de la baleine, le dégraissage est réalisé dans des bains de solvants organiques. Les solvants utilisés sont des alcools (méthanol, éthanol), des aldéhydes (formol), des cétones (acétone, méthyléthylcétone) et des solvants chlorés (trichloréthane, trichloréthylène, perchloréthylène). En effet, les lipides ont une solubilité nulle ou faible dans l'eau mais élevée dans les solvants organiques peu polaires.

\section{Limites et inconvénients de ces méthodes traditionnelles}

Ces différents procédés de nettoyage ne sont pas sans effet sur l'intégrité du matériau. Ils peuvent entrâner une altération des constituants de l'os (modifications des protéines mais aussi de la fraction minérale) qui fausse toute étude scientifique ultérieure (anatomie comparée, taxonomie, analyse d'ADN, pathologie, taphonomie, histologie...) et des déformations qui rendent difficile le remontage du squelette.

Les traitements en milieux aqueux ont une efficacité limitée à cause de la faible solubilité des graisses dans l'eau. D'après S. Williams, l'eau n'occasionne pas d'altérations sérieuses mais les additifs présents en solution aqueuse peuvent dégrader la partie organique ou minérale de l'os, que ce soit des additifs involontaires (sels minéraux) ou volontaires (oxydant 

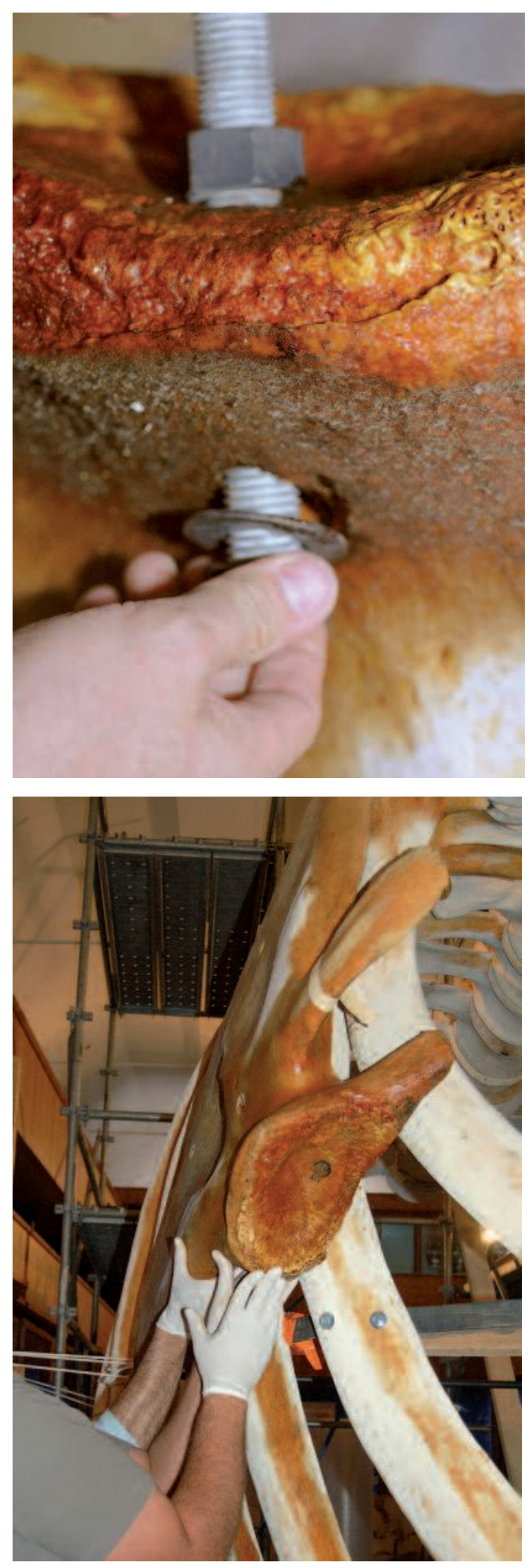

Omoplates du rorqual en cours de remontage (clichés 2008) : non seulement la graisse modifie l'aspect de surface de l'os, mais elle l'expose aussi à des dégradations physico-chimiques et biologiques. Au niveau des perforations de montage ostéologique, la présence de graisse au cœur de l'os est visible. (c) Arc'Antique/J.G. Aubert et ๑) Muséum d'Histoire naturelle de Nantes/Patrick Jean
$\left.\mathrm{H}_{2} \mathrm{O}_{2}\right)$ ou base $(\mathrm{NaOH}, \mathrm{KOH})$ ). Les milieux basiques favorisent la dégradation des graisses mais dénaturent également les protéines. Les enzymes actuellement ajoutées à la macération (papaïne, neutrase) influencent peu le dégraissage car il s'agit de protéases qui agissent principalement sur les protéines (étape d'écharnage) et non sur les lipides.

S. Williams note également l'effet désastreux des températures élevées généralement associées aux traitements aqueux : au-delà de $45^{\circ} \mathrm{C}$, les fibres de collagène sont dégradées. La bonne conservation des os nécessite un maintien de l'humidité relative (HR) entre 45 et $60 \%$. Un mauvais séchage après un traitement aqueux peut altérer l'os : un séchage trop important implique une dessiccation de l'os qui peut alors se fissurer et un séchage insuffisant occasionne un développement de microorganismes.

Les traitements à base de solvants organiques peuvent aussi provoquer un assèchement du matériau et le fragiliser. De plus, l'utilisation des solvants chlorés favorise l'acidification du matériau en formant de l'acide chlorhydrique en présence d'humidité. Y. Fernàndez-Jalvo observe une destruction de la couche superficielle de l'os en milieu acide. Les analyses n'ont pas été menées au cœur du matériau, mais les altérations sont certainement présentes dans l'ensemble de l'os.

Parmi toutes les techniques actuelles de dégraissage, l'utilisation des solvants organiques est certainement la plus efficace mais pas sans conséquence sur la conservation de l'os. De plus, ces produits chimiques présentent divers inconvénients, exacerbés lors des traitements des squelettes de grandes dimensions, comme ceux du rorqual : inflammabilité du produit (acétone, éthanol), nocivité pour l'homme, causant irritations et brûlures par contact avec la peau ou les yeux (acétone), toxicité par inhalation (trichloréthane, trichloréthylène) voire effet cancérogène (trichloréthylène). Ils ont également un impact néfaste sur l'environnement (solvants chlorés en particulier), ce qui implique une prise en compte de leur recyclage lors de leur utilisation; la réglementation sur les solvants chlorés s'est d'ailleurs fortement durcie ces dernières années.

\section{Alternatives explorées : avantages et limites}

Le laboratoire Arc'Antique s'est donc interrogé sur la possibilité d'avoir recours à des alternatives pour extraire les graisses résiduelles du squelette de rorqual exposé au muséum d'Histoire naturelle de Nantes. Les solutions envisagées par Arc'Antique 


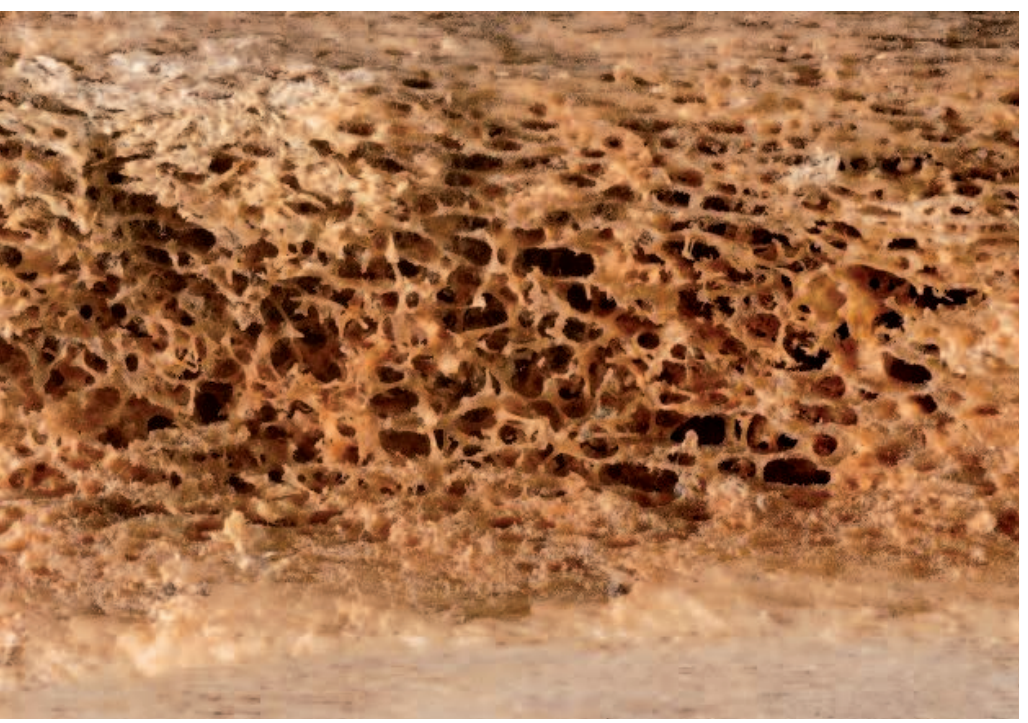

Dans la structure de l'os, sous une couche externe dense et compacte, le périoste (en bas du cliché), la partie interne de

I'os ou spongiosa est très poreuse et gorgée de graisse. ( ) Arc'Antique/J.-G. Aubert

pour augmenter la solubilité des graisses et faciliter leur extraction sont :

- l'utilisation d'enzymes en milieux aqueux ou semi aqueux (eau / éthanol),

- le $\mathrm{CO}_{2}$ supercritique comme solvant,

- les solvants végétaux.

\section{Les enzymes}

Les enzymes, telles que les lipases, permettent de dégrader les triglycérides en acides gras, les acides gras étant plus solubles que les triglycérides en milieu aqueux. Des traitements de dégraissage aux lipases ont déjà été menés sur des squelettes de cétacés, notamment au Musée canadien de la Nature (12) ; au Danemark, The School of Conservation de Copenhague entreprend un projet similaire au nôtre (13). Dans le but de comprendre et maîtriser le fonctionnement des lipases afin d'améliorer leur action lors du traitement, Arc'Antique a développé un partenariat avec l'équipe Génie Enzymatique (Laboratoire d'applications des lipases) de l'IUT Génie Biologique de Laval, université du Maine.

L'intérêt des enzymes réside dans leur grande sélectivité d'action, elles paraissent donc idéales dans une démarche de conservation-restauration dans la mesure où elles s'attaquent uniquement aux graisses, le collagène et l'apatite sont ainsi préservés. Par contre, leur utilisation nécessite des conditions strictes de concentration, de température, de $\mathrm{pH}$. Néanmoins, ce procédé n'est envisageable que sur des graisses peu dégradées et n'est donc pas applicable, a priori, aux squelettes anciennement préparés et exposés depuis des années dans des conditions ambiantes, ce qui est le cas du rorqual du muséum de Nantes. Il est cependant adapté aux spécimens frais, récemment décharnés. L'efficacité de ce procédé au cœur de l'os reste cependant à définir.

\section{Le $\mathrm{CO}_{2}$ supercritique}

Les capacités dégraissantes du $\mathrm{CO}_{2}$ supercritique (14) sont exploitées dans le nettoyage industriel de pièces métalliques en cours d'usinage. Les triglycérides sont en effet solubles dans le $\mathrm{CO}_{2}$ supercritique : solubilité de $1 \%$ à 250-300 bar et à $35-40^{\circ} \mathrm{C}$, qui sélève à $10 \%$ en présence d'un co-solvant, tel que l'éthanol. Par contre, les protéines sont insolubles dans le $\mathrm{CO}_{2}$ supercritique. Ce procédé, dit " propre ", est donc très prometteur pour le dégraissage d'os et a d'ailleurs déjà été utilisé avec succès pour la délipidation des greffons osseux. Mais pour notre application, plusieurs problèmes se posent. Le premier réside dans la taille limitée des enceintes de traitement $(2 \mathrm{~m} 70 \mathrm{x}$ diamètre $50 \mathrm{~cm}$ ) ; les appareillages actuellement utilisés en industrie ne permettent pas de traiter la majorité des os du squelette de rorqual. D’autre part, une étude préalable doit être menée pour évaluer la pénétration du solvant dans les objets de cette taille et pour vérifier la tenue mécanique des os lors des variations de pression.

\section{Les solvants végétaux}

Les solvants végétaux, dits biosolvants, possèdent les mêmes fonctionnalités que les solvants organiques. Ils proviennent d'amidon, d'oléagineux, ou d'autres végétaux tels que les agrumes, le pin, le colza, le lin, l'olivier... Ils sont déjà couramment utilisés comme dégraissants. Il reste à définir quel type de biosolvant serait le plus efficace pour solubiliser les graisses présentes dans les os du squelette de rorqual. Une étude doit être réalisée au préalable pour évaluer la pénétration de ces produits au cœur de l'os. Il faudra aussi s'assurer de la possibilité d'éliminer ces biosolvants des os en fin de traitement. Les interactions entre ces produits et les éléments constitutifs de l'os (collagène et apatite) devront aussi être examinées.

\section{Bilan et perspectives}

La problématique du dégraissage des squelettes, à travers le cas de la baleine du muséum de Nantes, est donc étendue et complexe. Étendue car présente dans de nombreuses collections anciennes ou récemment préparées de muséums d'Histoire naturelle. Complexe par la nature des matériaux en présence et 


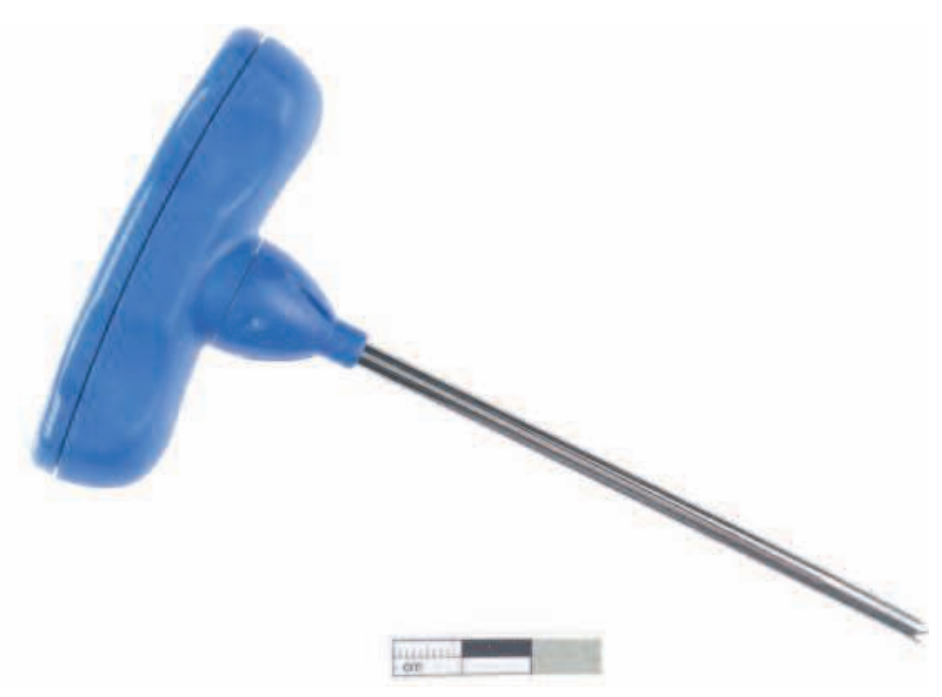

Le trocart, outil médical, permet de réaliser au cœur de l'os des prélèvements discrets $(3 \mathrm{~mm}$ de diamètre) sans dénaturer l'échantillon à analyser. (c) Arc'Antique/J.-G. Aubert

le volume conséquent de l'espèce à traiter. Les méthodes de dégraissage aujourd'hui employées présentent toutes des limites : toxicité, altération du matériau ou inefficacité sur les graisses.

La pratique du dégraissage de squelettes requiert une connaissance pointue des propriétés physicochimiques du substrat à traiter, l'os gorgé de graisse, des interactions entre l'os et les produits de dégraissage employés, mais aussi la compréhension des effets des paramètres de traitement.

Un diagnostic préalable à toute intervention s'avère indispensable ; on estimera l'état de conservation de l'os et sa teneur en graisse, le degré d'altération des graisses en surface et à cœur de l'os. Bien sûr on ne peut se fier uniquement à l'aspect visuel de l'os, à son état de surface : une surface propre et blanche n'implique pas forcément un os suffisamment dégraissé à cœur. Le diagnostic s'appuiera donc sur des analyses de l'os gras prélevé au scalpel sur la surface et à cour par carottage. La graisse extraite de l'échantillon réduit en poudre peut être identifiée par chromatographie en couche mince (détermination des grandes familles chimiques). La composition précise en acides gras est obtenue par chromatographie en phase gazeuse (HPGC).

Des essais menés par Arc'Antique ont permis de déterminer une méthode de prélèvement au trocart, outil utilisé en médecine, qui présente l'avantage de ne pas chauffer l'échantillon et d'éviter ainsi toute modification des graisses. Le prélèvement est discret, le diamètre extérieur du trocart étant de 4 mm ; la perforation réalisée peut ensuite être aisément masquée par un comblement mis en teinte (15). Il est également possible de réaliser les prélèvements aux endroits où l'on prévoit des perforations de montage ostéologique ultérieur (16).

Si un traitement de dégraissage est entrepris, il devra être contrôlé par analyses pour s'assurer de son efficacité à cœur et de la non-altération de l'os. La spectrométrie Raman, effectuée directement sur la carotte, évalue la proportion de collagène et d'apatite : elle est donc révélatrice de l'altération des os. La carotte, non détruite par ce type d'analyse, peut ensuite être remise en place.

Avant toute intervention, des mesures du $\mathrm{pH}$ sont nécessaires pour évaluer l'acidité éventuelle de l'os, qui constitue un danger pour la conservation de sa partie minérale. Cette acidité peut être due notamment à la présence d'acide chlorhydrique formé en présence d'humidité à la suite d'un traitement au solvant chloré, comme c'est le cas pour le rorqual du muséum de Nantes.

Il est important de prendre en compte les risques qu'implique, après 13 ans d'exposition et donc de relative stabilisation dans les conditions environnementales, un nouveau traitement sur le squelette du muséum de Nantes. Les recherches sont donc à poursuivre dans ce domaine. Des études similaires sont en cours au Danemark et Canada. Une mise en commun des connaissances et expériences permettrait peut-être de trouver un traitement adapté, une alternative aux solvants organiques toxiques. Dans l'attente de cette issue, on privilégiera la conservation préventive du squelette insuffisamment dégraissé dans des conditions hygrométriques adéquates.

Les auteurs tiennent à remercier Pierre Watelet (directeur du muséum d'Histoire naturelle de Nantes), Laurent Poisson et Françoise Ergan (équipe Génie Enzymatique, IUT Génie Biologique de Laval, université du Maine), Sabine Le Blond (étudiante IUT Chimie Rouen) et Claire Musso (étudiante Master de Conservation-Restauration des Biens culturels, université Paris I Panthéon-Sorbonne).

\section{Notes}

(1) Allen, N., Edge, M. et Horie, C. Polymers in Conservation. Royal Society of Chemistry, 1991, pp.39-41.

(2) Le Blond, S. Mise en place d'un protocole de suivi du dégraissage d'un squelette de baleine. Rapport de stage Arc'Antique/IUT de Chimie de Rouen, 2008, $50 \mathrm{p}$. 
(3) Le Blond, S., Guilminot, E., Lemoine, G., Huet, N. et Mevellec J.-Y. FT-Raman spectroscop : a positive mean of evaluating the impact of whale bone preservation treatment, article soumis à Vibrational Spectroscopy Manuscript Draft, 2009.

(4) Musso, C. Documentation et mise au point de tests pour le dégraissage d'un squelette de baleine. Rapport de stage Arc'Antique/Master 1 de Conservation-Restauration des Biens culturels, université Paris I Panthéon-Sorbonne, 2008, 88 p.

(5) Reiche, I., Chadefaux, Vignaud, C., et Menu, M. Les matériaux osseux archéologiques. Des biomatériaux nanocomposites complexes, L'actualité chimique, n³12-313, octobre-novembre 2007, pp. 86-92.

(6) Williams, S. Destructive preservation : a review of the Effect of Standard Preservation Practices on the Future Use of Natural History Collections. Göteborg : Studies in Conservation $n^{\circ} 6$, Acta Universitatis Gothoburgensis, 1999, pp. 23-35.

(7) Les triglycérides sont des triesters d'acides gras : ils résultent de la condensation d'acides gras avec un triol (trois fonctions alcool), le glycérol, par des liaisons esters.

(8) Échantillon d’huile prélevé en 1991 lors de l'échouage du rorqual : l'huile constitue un échantillon « natif » (non dégradé car conservé en tube fermé) ; les autres prélèvements, dépôts de matière brune sur une nageoire pectorale (membre supérieur) non dégraissée, graisse prélevée sur une côte et sur une omoplate dégraissées et exposées, constituent des échantillons « dégradés ».

(9) À ce jour seules les graisses prélevées en surface ont été identifiées ; restent à analyser des prélèvements d'os gras à cœur pour s'assurer que l'état de dégradation des lipides est le même qu'en surface.
(10) Fernàndez-Jalvo, Y. et Monfort, M.-D.-M. Experimental taphonomy in museums : Preparation protocols for skeletons and fossil vertebrates under the scanning electron microscopy, Geobios, n41, 2008, pp. 157181.

(11) Carter, D. et Walker A. Osteological preparation techniques, Care $\&$ Conservation of natural history collections, Ed. Butterworth-Heinemann, Oxford, 1999, pp. 9-14.

(12) Communication personnelle de Clayton Kennedy, Sr Collection Technician, Earth Sciences Section, Canadian Museum of Nature.

(13) Communication personnelle de Kim Simonsen, Associate Professor, Academy's School of Conservation, Denmark.

(14) Un fluide est dit supercritique lorsqu'il est placé dans des conditions de température et de pression au-delà de son point critique ; il est alors dans un état supercritique intermédiaire entre les états liquides et gazeux lui conférant les propriétés d'un solvant. Le fluide obtenu est caractérisé par une grande diffusivité (de l'ordre de celle des gaz) et une densité élevée proche de celle des liquides qui le dote d'une capacité de transport et d'extraction importante.

(15) Le comblement sera réalisé avec des matériaux stables dans le temps et réversibles, en accord avec les principes de déontologie de la conservation-restauration.

(16) Il est d'ailleurs à noter que dans les os longs, les épiphyses (ou extrêmités) sont les parties les plus grasses (communication personnelle de Patrick Costiou, professeur d'Anatomie à l'École vétérinaire de Nantes, ayant dirigé la préparation du squelette dans les années 1990). 